\title{
Adhesion molecule profiles of B-cell non-Hodgkin's lymphomas in the leukemic phase
}

D.M. Matos, E.G. Rizzatti,

A.B. Garcia, D.A.P. Gallo and R.P. Falcão
Departamento de Clínica Médica e Centro de Terapia Celular, Faculdade de Medicina de Ribeirão Preto, Universidade de São Paulo, Ribeirão Preto, SP, Brasil
Correspondence

R.P. Falcão

Laboratório de Hematologia

Departamento de Clínica Médica

FMRP, USP

Av. Bandeirantes, 3900

14049-900 Ribeirão Preto, SP

Brasil

Fax: +55-16-3633-1144

E-mail: rpfalcao@fmrp.usp.br

Publication supported by FAPESP.

.....................

Received May 9, 2006

Accepted August 15, 2006

\begin{abstract}
We evaluated the expression of 10 adhesion molecules on peripheral blood tumor cells of 17 patients with chronic lymphocytic leukemia, 17 with mantle-cell lymphoma, and 13 with nodal or splenic marginal B-cell lymphoma, all in the leukemic phase and before the beginning of any therapy. The diagnosis of B-cell non-Hodgkin's lymphomas was based on cytological, histological, immunophenotypic, and molecular biology methods. The mean fluorescence intensity of the adhesion molecules in tumor cells was measured by flow cytometry of CD19-positive cells and differed amongst the types of lymphomas. Comparison of chronic lymphocytic leukemia and mantle-cell lymphoma showed that the former presented a higher expression of CD11c and CD49c, and a lower expression of CD11b and CD49d adhesion molecules. Comparison of chronic lymphocytic leukemia and marginal B-cell lymphoma showed that the former presented a higher expression of CD49c and a lower expression of CD11a, CD11b, CD18, CD49d, CD29, and CD54. Finally, comparison of mantle-cell lymphoma and marginal B-cell lymphoma showed that marginal B-cell lymphoma had a higher expression of CD11a, CD11c, CD18, CD29, and CD54. Thus, the CD49c/CD49d pair consistently demonstrated a distinct pattern of expression in chronic lymphocytic leukemia compared with mantle-cell lymphoma and marginal B-cell lymphoma, which could be helpful for the differential diagnosis. Moreover, the distinct profiles of adhesion molecules in these diseases may be responsible for their different capacities to invade the blood stream.
\end{abstract}

\section{Introduction}

B-cell non-Hodgkin's lymphomas (BNHL) are a heterogeneous group of neoplastic diseases whose tumor cells are the malignant counterparts of normal B-lymphocytes (1). Although in most cases B-NHL arise from the lymph node (2), they can also display a leukemic phase characterized by the
Key words

- Non-Hodgkin's lymphoma

- Adhesion molecules

- Flow cytometry

- CD49c/CD49d presence of lymphoma cells in peripheral blood. The frequency of patients in the leukemic phase is different among the subtypes of B-NHL: in chronic lymphocytic leukemia, by definition, the involvement of peripheral blood is present in all patients (3); in mantle-cell lymphoma, the leukemic phase is present in $26-44 \%$ of cases $(4,5)$, but has also been reported to be present in $77 \%$ of 
cases in other series (6), and in nodal and splenic marginal B-cell lymphoma, it has been reported to be present in 11 and $57 \%$ of cases, respectively (7). These differences in the clinical behavior of B-NHL are most likely related to differential expression of various adhesion molecules on the membrane of tumor cells (4).

Adhesion molecules are a group of ligands and receptors involved in several biological processes, particularly cell migration (8). Given that some B-NHL subtypes are considered to be the malignant counterparts of distinctive steps in normal B-cell development, it is reasonable to infer that the malignant lymphoid cells use the same mechanisms of lymphocyte migration as normal Bcells to disseminate from their primary sites. In fact, a number of in vitro (9) and preclinical experiments $(10,11)$, as well as clinical observations (12-14) give support to this hypothesis.

Here we studied the expression of 10 adhesion molecules in tumor cells from the peripheral blood of patients with leukemic B-NHL (chronic lymphocytic leukemia, mantle-cell lymphoma, nodal or splenic marginal B-cell lymphoma) to determine how the down-regulation of specific adhesion markers inherently associated with malignant transformation contributes to the biogenesis of the leukemic phase observed in some B-NHL subtypes.

\section{Patients, Material and Methods}

\section{Patients}

We studied the peripheral blood of 47 patients with a diagnosis of B-NHL in the leukemic phase: 17 with chronic lymphocytic leukemia, 17 with mantle-cell lymphoma, and 13 with nodal or splenic marginal B-cell lymphoma. Some clinical and laboratory features of the patients are shown in Table 1. We defined the leukemic phase of B-NHL by the presence of a minimum of
3000 abnormal lymphoid cells/ $\mu \mathrm{L}$ associated with immunoglobulin light chain restriction. The flow cytometry studies were performed before the beginning of treatment. The research protocol was approved by the Ethics Committee of the University Hospital, School of Medicine of Ribeirão Preto, and written informed consent was obtained from all patients.

The diagnosis of B-NHL was based on cytological, histological, immunophenotypic, and molecular biology methods. All cases of chronic lymphocytic leukemia had a score of 4 or 5 in the Matutes scoring system $(15,16)$, while mantle-cell lymphoma and marginal B-cell lymphoma had a score of 0 to 3. In addition, except for one patient, who showed CYCLIN D1 positivity only by immunohistochemistry, all cases of mantlecell lymphoma showed evidence of CYCLIN Dl overexpression as measured by real-time polymerase chain reaction (data not shown).

\section{Flow cytometry}

All samples were analyzed with a FACScan flow cytometer (Becton Dickinson, San Jose, CA, USA) equipped with an argon ion laser with a wavelength of $488 \mathrm{~nm}$, by collecting 10,000 events per tube. The Cell Quest software (Becton Dickinson) was used for data acquisition and analysis. Only CD19-positive cells were gated for analysis (Figure 1A), and the expression of adhesion molecules was measured by the mean fluorescent intensity (MFI) obtained from histogram graphs on a linear scale (Figure 1B). Since the setting of an arbitrary cut-off value led to no meaningful conclusions, we preferred to use the MFI as the variable to estimate the intensity of expression of the adhesion molecules. The final MFI value was obtained by subtracting the "MFI of non-specific isotypes" from the "MFI of the relevant antigen".

Mononuclear cells were isolated from peripheral blood samples by Fycoll Hypaque 
density gradient centrifugation $(1.077 \mathrm{~g} / \mathrm{mL}$; Becton Dickinson). The flow cytometry panel consisted of monoclonal antibodies against the following adhesion molecules (with the respective clones in parentheses): CD11a, $\alpha_{L}$ integrin (HI111); CD11b, $\alpha_{M}$ integrin (ICRF44); CD11c, $\alpha_{\mathrm{X}}$ integrin (B-ly6); CD18, $\beta_{2}$ integrin (6.7); CD29, $\beta_{1}$ integrin (MAR4); CD44, H-CAM (G44-26); CD49c, $\alpha_{3}$ integrin, VLA-3 (C3II.1); CD49d, $\alpha_{4}$ integrin, VLA-4 (9F10); CD54, ICAM-1 (HA58); CD62L, L-selectin (Dreg-56). All monoclonal antibodies were conjugated with fluorescein isothiocyanate, phycoerythrin, or perydin chlorophyl protein and all were purchased from Becton Dickinson. To exclude $\mathrm{T}$ lymphocytes and monocytes from analysis, CD19 was added to all tubes: tube 1 , CD18 x CD11a x CD19; tube 2, CD11b x CD19; tube 3, CD11c x CD19; tube 4, CD44 x CD29 x CD19; tube 5, CD62 x CD54 x CD19; tube 6, CD49c x CD19; tube 7, CD49d x CD19.

\section{Statistical analysis}

Comparison of the expression of adhesion molecules between the three groups was performed by the Kruskal-Wallis test using GraphPad Prism and Statistical Analysis System (SAS) softwares, with the level of significance set at $\mathrm{P}<0.05$. When a $\mathrm{P}$ value of 0.05 or less was found in the Kruskal-Wallis test, the Dunn post-test was applied to analyze pairs of groups.

\section{Results}

The expression of the adhesion molecules of malignant lymphoid cells was measured by flow cytometry in CD19-positive cells. The MFI of the adhesion molecules CD11a, CD11b, CD11c, CD18, CD49c, CD49d, CD29, and CD54 was different in the three groups, as shown in Table 2. The Dunn posttest was applied when the $\mathrm{P}$ value was 0.05 or less. The results of pair comparisons are
Table 1. Clinical and laboratory features of the patients according to diagnosis.

\begin{tabular}{lccc}
\hline & CLL & MCL & MZL \\
\hline Sex (M/F) & $11 / 6$ & $14 / 3$ & $10 / 3$ \\
Age (years) & $62(45-78)$ & $67(34-81)$ & $66(28-95)$ \\
Lymphadenomegaly & $9 / 17(53 \%)$ & $11 / 17(65 \%)$ & $4 / 13(31 \%)$ \\
Splenomegaly & $8 / 17(47 \%)$ & $14 / 17(82 \%)$ & $13 / 13(100 \%)$ \\
Lymphocytes (x 109/L) & $42.7(6.0-122.4)$ & $62.4(6.7-256.0)$ & $18.7(3.6-67.6)$
\end{tabular}

Data are reported as median (range) or number (percent). CLL = chronic lymphocytic leukemia; $\mathrm{MCL}=$ mantle-cell lymphoma; $\mathrm{MZL}=$ marginal $\mathrm{B}$-cell lymphoma.
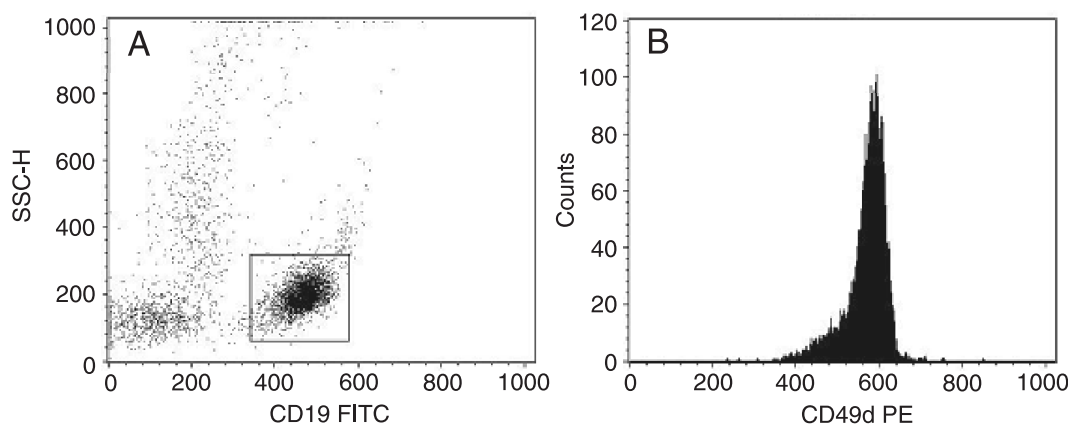

Figure 1. Flow cytometry graphs. $A, A$ gate strategy graph: only both CD19-positive and low side scatter-SSC (internal cellular granularity) cells were selected for analysis (square). $B$, A histogram graph: a single-parameter histogram in linear scale shows the expression of the adhesion molecule CD49d.

Table 2. Adhesion molecule on lymphoma cells according to diagnosis.

\begin{tabular}{lccc}
\hline & CLL $(\mathrm{N}=17)$ & $\mathrm{MCL}(\mathrm{N}=17)$ & $\mathrm{MZL}(\mathrm{N}=13)$ \\
\hline CD11a & $167.9(20.7-376.9)$ & $257.9(90.2-363.5)$ & $401.6(203-519.4)$ \\
CD11b & $0 \quad(0-53.2)$ & $21.7(0-274.1)$ & $42.7(0-409.3)$ \\
CD11c & $46.3(0-222.7)$ & $0 \quad(0-43.5)$ & $143.6(0-425.2)$ \\
CD18 & $105.9(15.5-264.4)$ & $150.1(52.2-244.7)$ & $275.2(194-415.9)$ \\
CD49c & $75.5(0-274.8)$ & $0(0-87.2)$ & 0 \\
CD49d & $97.9(0-369.8)$ & $248.0(135.9-430.4)$ & $362.1(196.3-443.7)$ \\
CD29 & $117.6(11.7-335.6)$ & $116.6(2.8-310)$ & $236.0(75.3-353.3)$ \\
CD44 & $311.4(175.3-422.8)$ & $316.4(228.4-431.7)$ & $285.0(84.1-383.7)$ \\
CD54 & $223.4(117.7-308)$ & $238.1(134.5-413.2)$ & $317.2(191.3-470.1)$ \\
CD62 & $4.2(0-98.4)$ & $10.2(0-108.6)$ & $12.1(0-302.7)$
\end{tabular}

Data are reported as median and range (in parentheses) of the mean fluorescent intensity (MFI). The MFI represents the mean fluorescence of all events (lymphoma cells) in the histogram graphs. $C L L=$ chronic lymphocytic leukemia; $M C L=$ mantle-cell Iymphoma; MZL = marginal B-cell lymphoma. The Kruskal-Wallis test gave $\mathrm{P}<0.05$ for all markers except CD44 and CD62, indicating that there was at least one significant difference between most of the markers for the three cell types. 
shown in Table 3.

Comparison of chronic lymphocytic leukemia and mantle-cell lymphoma showed that chronic lymphocytic leukemia presented a higher expression of CD11c and CD49c, and a lower expression of CD11b and CD49d. Comparison of chronic lymphocytic leukemia and marginal B-cell lymphoma showed that the former presented a higher expression of CD49c and a lower expression of CD11a, CD11b, CD18, CD49d, CD29, and CD54. Finally, comparison of mantle-cell lymphoma and marginal B-cell lymphoma showed that the latter had a higher expression of CD11a, CD11c, CD18, CD29, and CD54.

\section{Discussion}

We analyzed the expression of 10 adhesion molecules in the peripheral blood of 47 patients with a diagnosis of B-NHL. We expected that the higher frequency of blood invasion by tumor cells in chronic lymphocytic leukemia, when compared with the other B-NHL, could be explained by specific profiles of adhesion molecule expression.

Our finding of the lower expression of

Table 3. Pairwise comparison of B-cell non-Hodgkin's lymphomas for surface antigens by the Dunn post-test.

CLL x MCL CLL x MZL MCL x MZL

\begin{tabular}{lccc}
\hline CD11a & NS & $S$ & $S$ \\
CD11b & $S$ & $S$ & NS \\
CD11c & $S$ & NS & $S$ \\
CD18 & NS & $S$ & $S$ \\
CD49c & $S$ & $S$ & $N S$ \\
CD49d & S & $S$ & $N S$ \\
CD29 & NS & $S$ & $S$ \\
CD44 & NA & NA & NA \\
CD54 & NS & S & S \\
CD62 & NA & NA & NA \\
\hline
\end{tabular}

$\mathrm{CLL}=$ chronic lymphocytic leukemia; $\mathrm{MCL}=$ mantle-cell lymphoma; MZL = marginal B-cell lymphoma; $\mathrm{S}=$ differences are significant $(\mathrm{P} \leq 0.05)$; $\mathrm{NS}=$ differences are nonsignificant; $\mathrm{NA}=$ not applicable.
CD11a and CD49d in chronic lymphocytic leukemia suggests that these adhesion molecules are probably responsible for the different compartment the disease infiltrates compared to its related entity, the small lymphocytic lymphoma $(17,18)$. Actually, since the structure of normal lymphoid follicles in lymph nodes depends on the appropriate association between B lymphocytes and dendritic follicular cells through the interaction of CD11a and ICAM-1 (an intercellular adhesion molecule), and of CD49d and VCAM1 (a vascular cell adhesion molecule), respectively (19), the lower expression of CD11a and CD49d on tumor cells of chronic lymphocytic leukemia could facilitate their detachment from the lymph node, while the expression of these adhesion molecules is preserved in small lymphocytic lymphoma $(17,18)$. In fact, the down-regulation of CD11a and CD49d in chronic lymphocytic leukemia could also explain the peripheral blood invasion by these malignant cells, as compared with the occasional infiltration observed in mantle-cell lymphoma and marginal B-cell lymphoma.

Our findings of a greater expression of CD49c and a lower expression of CD49d in chronic lymphocytic leukemia as compared with the other B-NHL have been previously observed $(20,21)$. The combination of strong expression of CD49c and weak expression of CD49d is the hallmark of chronic lymphocytic leukemia and can be used in the differentiation between this disease and the other B-NHL in the leukemic phase. In fact, it has been shown that imbalance in CD49c/ CD49d expression in chronic lymphocytic leukemia also contributes to the peripheral blood invasion (22). Moreover, a recent study has demonstrated prognostic relevance for the combination of high CD49c and low CD49d expression in chronic lymphocytic leukemia (23).

CD29 is the common $\beta 1$ chain of $\alpha$ subunits of integrins $\alpha 3 / \mathrm{CD} 49 \mathrm{c}$ and $\alpha 4 / \mathrm{CD} 49 \mathrm{~d}$ (8). Since the CD29 and $\alpha$ subunits are 
expressed together by a non-covalent bond, the higher expression of this adhesion molecule observed in marginal B-cell lymphoma is probably the result of the concomitant higher expression of CD49d in this subtype of B-NHL.

The presence of the myelomonocytic antigens CD11b and CD11c on cells of chronic lymphocytic leukemia has been previously reported $(24,25)$. CD11b has a heterogeneous expression in chronic lymphocytic leukemia. Whereas some publications described a high expression of this adhesion molecule in chronic lymphocytic leukemia (20), others reported a low expression $(21,25)$. The data presented in the current study are in agreement with these latter reports, and since the mantle-cell lymphoma and the marginal B-cell lymphoma showed a higher expression of $\mathrm{CD} 11 \mathrm{~b}$ compared to chronic lymphocytic leukemia, this adhesion molecule could eventually be used to help differentiate between chronic lymphocytic leukemia and the other B-NHL in the leukemic phase. Moreover, CD11b may have other biological functions in chronic lymphocytic leukemia since it has been reported to be associated with a higher probability of disease progression and poor survival (26), as well as to prevent the induction of apoptosis in this disease (27). Our findings add more information about the expression of $\mathrm{CD} 11 \mathrm{~b}$ in mantle-cell lymphoma and in marginal B-cell lymphoma since there are only a few studies in the literature regarding this adhesion molecule in these diseases (28).

The role of CD11c in the dissemination of B-NHL is still controversial. Angelopoulou et al. (28) and Bairey et al. (29) found a higher frequency of splenic involvement in cases of chronic lymphocytic leukemia with strong CD11c positivity. Moreover, this adhesion molecule is usually over-expressed in splenic marginal B-cell lymphoma (28) and in hairy cell leukemia $(30,31)$, both diseases characterized by large splenomegaly, which could suggest a role for CD11c in the infiltration of the spleen. However, our data do not support this hypothesis, since 14 of 17 of our patients with the diagnosis of mantle-cell lymphoma presented an enlarged spleen, but showed a very low expression of CD11c. In agreement with our results, Angelopoulou et al. (32), in a study of 11 patients with mantle-cell lymphoma, observed that the CD11c was negative in all patients, including a subgroup of 6 patients with exclusively splenic disease.

CD54 is a member of the immunoglobulin superfamily that normally has a lower expression in peripheral blood mononuclear cells when compared with the lymph node (33). In our series, marginal B-cell lymphoma presented the highest expression of this adhesion molecule. Similarly, Csanaky et al. (34), studying 7 patients with this disease, found a higher level of CD54 expression when compared with chronic lymphocytic leukemia. Stauder et al. (35) and Horst et al. (36) showed that the lower expression of CD54 in indolent B-NHL correlates with a higher frequency of invasion of peripheral blood. In addition, Terol et al. (37) showed that low expression or absence of CD54 was associated with a higher frequency of disseminated disease (stage IV), extranodal involvement and bone marrow infiltration. Down-regulation of CD54 seems to favor the dissemination of tumor cells by a still unknown biological pathway and little information is available about the expression of this adhesion molecule in splenic marginal B-cell lymphoma.

The results of the present study allow us to conclude that chronic lymphocytic leukemia, mantle-cell lymphoma and marginal B-cell lymphoma have different adhesion molecule profiles, and these differences may be responsible for the distinct capacities of these diseases to disseminate into the blood stream. Moreover, our data reinforce the hypothesis that the peripheral blood invasion always present in chronic lymphocytic leukemia is the consequence of an imbalance of adhesion 
molecule expression in tumor cells. Furthermore, we demonstrated that the CD49c/CD49d pair consistently presented a distinct pattern of expression in chronic lymphocytic leukemia compared to mantle-cell lymphoma and marginal B-cell lymphoma, which could be help- ful for the differential diagnosis.

Future studies comparing the expression of adhesion molecules in peripheral blood and lymph nodes could better address their role in the phenomenon of peripheral blood invasion in B-NHL.

\section{References}

1. Kuppers R. Mechanisms of B-cell lymphoma pathogenesis. Nat Rev Cancer 2005; 5: 251-262.

2. Muller AM, Ihorst G, Mertelsmann R, Engelhardt M. Epidemiology of non-Hodgkin's lymphoma (NHL): trends, geographic distribution, and etiology. Ann Hematol 2005; 84: 1-12.

3. Müller-Hermelink HK, Montserrat E, Catovsky D, Harris NL. Chronic lymphocytic leukemia/small lymphocytic lymphoma. In: Jaffe ES, Harris NL, Stein H, Vardiman JW (Editors), World Health Organization Classification of Tumours. Pathology and genetics of tumours of haematopoietic and lymphoid tissues. Lyon: IARC Press; 2001. p 127-130.

4. Criel A, Pittaluga S, Verhoef G, Wlodarska I, Meeus P, Mecucci C, et al. Small B cell NHL and their leukemic counterpart: differences in subtyping and assessment of leukemic spread. Leukemia 1996; 10 : 848-853.

5. Bain BJ, Catovsky D. The leukaemic phase of non-Hodgkin's lymphoma. J Clin Pathol 1995; 48: 189-193.

6. Cohen PL, Kurtin PJ, Donovan KA, Hanson CA. Bone marrow and peripheral blood involvement in mantle cell lymphoma. $\mathrm{Br} \mathrm{J}$ Haematol 1998; 101: 302-310.

7. Berger F, Felman $P$, Thieblemont $C$, Pradier $T$, Baseggio L, Bryon PA, et al. Non-MALT marginal zone B-cell lymphomas: a description of clinical presentation and outcome in 124 patients. Blood 2000; 95: 1950-1956.

8. Drillenburg P, Pals ST. Cell adhesion receptors in lymphoma dissemination. Blood 2000; 95: 1900-1910.

9. Stauder R, Hamader S, Fasching B, Kemmler G, Thaler J, Huber H. Adhesion to high endothelial venules: a model for dissemination mechanisms in non-Hodgkin's lymphoma. Blood 1993; 82: 262-267.

10. Roos E. Adhesion molecules in lymphoma metastasis. Cancer Metastasis Rev 1991; 10: 33-48.

11. Roossien FF, de Rijk D, Bikker A, Roos E. Involvement of LFA-1 in lymphoma invasion and metastasis demonstrated with LFA-1-deficient mutants. J Cell Biol 1989; 108: 1979-1985.

12. Pals ST, Drillenburg P, Radaszkiewicz T, Manten-Horst E. Adhesion molecules in the dissemination of non-Hodgkin's lymphomas. Acta Haematol 1997; 97: 73-80.

13. Freedman AS. Expression and function of adhesion receptors on normal B cells and B cell non-Hodgkin's lymphomas. Semin Hematol 1993; 30: 318-328.

14. Timens W. Cell adhesion molecule expression and homing of hematologic malignancies. Crit Rev Oncol Hematol 1995; 19: 111-129.

15. Matutes E, Owusu-Ankomah K, Morilla R, Garcia MJ, Houlihan A, Que TH, et al. The immunological profile of B-cell disorders and proposal of a scoring system for the diagnosis of CLL. Leukemia 1994; 8: 1640-1645.

16. Moreau EJ, Matutes E, A'Hern RP, Morilla AM, Morilla RM, Owusu-
Ankomah KA, et al. Improvement of the chronic lymphocytic leukemia scoring system with the monoclonal antibody SN8 (CD79b). Am J Clin Pathol 1997; 108: 378-382.

17. Inghirami G, Wieczorek R, Zhu BY, Silber R, la-Favera R, Knowles DM. Differential expression of LFA-1 molecules in non-Hodgkin's lymphoma and lymphoid leukemia. Blood 1988; 72: 1431-1434.

18. Nadkarni JJ, Perambakam SM, Rathore VB, Amin KM, Parikh PM, Naresh KN, et al. Expression of adhesion molecules in B-cell chronic lymphocytic leukaemia: an analysis in lymphoid compartments peripheral blood, bone marrow and lymph node. Cancer Biother Radiopharm 1998; 13: 269-274.

19. Koopman G, Parmentier HK, Schuurman HJ, Newman W, Meijer CJ, Pals ST. Adhesion of human B cells to follicular dendritic cells involves both the lymphocyte function-associated antigen $1 /$ intercellular adhesion molecule 1 and very late antigen 4/vascular cell adhesion molecule 1 pathways. J Exp Med 1991; 173: 1297-1304.

20. Baldini L, Cro L, Calori R, Nobili L, Silvestris I, Maiolo AT. Differential expression of very late activation antigen-3 (VLA-3)/VLA-4 in B-cell non-Hodgkin lymphoma and B-cell chronic lymphocytic leukemia. Blood 1992; 79: 2688-2693.

21. Sembries S, Pahl H, Stilgenbauer S, Dohner H, Schriever F. Reduced expression of adhesion molecules and cell signaling receptors by chronic lymphocytic leukemia cells with $11 \mathrm{q}$ deletion. Blood 1999; 93: 624-631.

22. Baldini LG, Cro LM. Structure and function of VLA integrins: differential expression in B-cell leukemia/lymphoma. Leuk Lymphoma 1994; 12: 197-203.

23. Zucchetto A, Sonego P, Degan M, Bomben R, Dal Bo M, Russo S, et al. Surface-antigen expression profiling (SEP) in B-cell chronic lymphocytic leukemia (B-CLL): Identification of markers with prognostic relevance. J Immunol Methods 2005; 305: 20-32.

24. De la Hera A, Alvarez-Mon M, Sanchez-Madrid F, Martinez C, Durantez A. Co-expression of Mac-1 and p150,95 on CD5+ B cells. Structural and functional characterization in a human chronic lymphocytic leukemia. Eur J Immunol 1988; 18: 1131-1134.

25. De Rossi G, Zarcone D, Mauro F, Cerruti G, Tenca C, Puccetti A, et al. Adhesion molecule expression on B-cell chronic lymphocytic leukemia cells: malignant cell phenotypes define distinct disease subsets. Blood 1993; 81: 2679-2687.

26. Tassies D, Montserrat E, Reverter JC, Villamor N, Rovira M, Rozman C. Myelomonocytic antigens in B-cell chronic lymphocytic leukemia. Leuk Res 1995; 19: 841-848.

27. Plate JM, Long BW, Kelkar SB. Role of beta2 integrins in the prevention of apoptosis induction in chronic lymphocytic leukemia B cells. Leukemia 2000; 14: 34-39.

28. Angelopoulou MK, Kontopidou FN, Pangalis GA. Adhesion molecules in B-chronic lymphoproliferative disorders. Semin Hematol 
1999; 36: 178-197.

29. Bairey O, Zimra Y, Rabizadeh E, Shaklai M. Expression of adhesion molecules on leukemic B cells from chronic lymphocytic leukemia patients with predominantly splenic manifestations. Isr Med Assoc J 2004; 6: 147-151.

30. Lucio PJ, Faria MT, Pinto AM, da Silva MR, Correia Junior ME, da Costa RJ, et al. Expression of adhesion molecules in chronic B-cell lymphoproliferative disorders. Haematologica 1998; 83: 104-111.

31. Marotta G, Raspadori D, Sestigiani C, Scalia G, Bigazzi C, Lauria F. Expression of the CD11C antigen in B-cell chronic lymphoproliferative disorders. Leuk Lymphoma 2000; 37: 145-149.

32. Angelopoulou MK, Kapiris E, Kourtis I, Siakantaris MP, Kittas Ch, Pangalis GA. The adhesion molecule profile of mantle cell lymphoma (MCL) vs B-chronic lymphocytic leukemia (B-CLL). Blood 1997; 90: 522 (Abstract).

33. Boyd AW, Dunn SM, Fecondo JV, Culvenor JG, Duhrsen U, Burns GF, et al. Regulation of expression of a human intercellular adhesion molecule (ICAM-1) during lymphohematopoietic differentiation. Blood 1989; 73: 1896-1903.
34. Csanaky G, Matutes E, Vass JA, Morilla R, Catovsky D. Adhesion receptors on peripheral blood leukemic $B$ cells. A comparative study on B cell chronic lymphocytic leukemia and related lymphoma/ leukemias. Leukemia 1997; 11: 408-415.

35. Stauder R, Greil R, Schulz TF, Thaler J, Gattringer C, Radaskiewicz $\mathrm{T}$, et al. Expression of leucocyte function-associated antigen-1 and 7F7-antigen, an adhesion molecule related to intercellular adhesion molecule-1 (ICAM-1) in non-Hodgkin lymphomas and leukaemias: possible influence on growth pattern and leukaemic behaviour. Clin Exp Immunol 1989; 77: 234-238.

36. Horst E, Radaszkiewicz T, Hooftman-den Otter A, Pieters R, van Dongen JJ, Meijer CJ, et al. Expression of the leucocyte integrin LFA-1 (CD11a/CD18) and its ligand ICAM-1 (CD54) in lymphoid malignancies is related to lineage derivation and stage of differentiation but not to tumor grade. Leukemia 1991; 5: 848-853.

37. Terol MJ, Lopez-Guillermo A, Bosch F, Villamor N, Cid MC, Rozman $\mathrm{C}$, et al. Expression of the adhesion molecule ICAM-1 in nonHodgkin's lymphoma: relationship with tumor dissemination and prognostic importance. J Clin Oncol 1998; 16: 35-40. 